\title{
Improving the Multiple Alignments Strategy for Fingerprint Verification
}

\author{
Miguel Angel Medina-Pérez ${ }^{1,2}$, Milton García-Borroto ${ }^{1}$, \\ Andres Eduardo Gutierrez-Rodríguez ${ }^{1}$, and Leopoldo Altamirano-Robles ${ }^{2}$ \\ 1 Centro de Bioplantas, Universidad de Ciego de Ávila. Carretera a Morón km 9, \\ Ciego de Ávila, Cuba, C.P. 69450 \\ \{migue, mil, andres\}@bioplantas.cu \\ 2 Instituto Nacional de Astrofísica, Óptica y Electrónica. Luis Enrique Erro No. 1, \\ Sta. María Tonanzintla, Puebla, México, C.P. 72840 \\ robles@inaoep.mx
}

\begin{abstract}
Developing accurate fingerprint verification algorithms is an active research area. A large amount of fingerprint verification algorithms are based on minutiae descriptors. An important component of these algorithms is the alignment strategy. The single alignment strategy, with $O\left(n^{2}\right)$ time complexity, uses the local matching minutiae pair that maximizes the similarity value to align the minutiae. Nevertheless, even if the selected minutiae pair is a true matching pair, it is not necessarily the best pair to carry out fingerprint alignment. The multiple alignments strategy alleviates these limitations by performing multiple minutiae alignments, increasing the time complexity to $O\left(n^{4}\right)$. In this paper, we improve the multiple alignment strategy, reducing its complexity while still achieving a high accuracy. The new strategy is based on the rationale that most minutiae descriptors from one fingerprint correspond with their most similar descriptors from the other fingerprint. To test the new strategy behavior, we adapt three well known algorithms to a traditional multiple alignment strategy and to our strategy. Several experiments in the FVC2004 database show that our strategy outperforms both the single and the multiple alignments strategies.
\end{abstract}

Keywords: biometrics, fingerprint verification, minutiae descriptor.

\section{Introduction}

Fingerprint recognition [10] is an active research area nowadays. It plays an important role in forensic applications, but its increasing popularity is a consequence of its integration into civilian systems. An important component in fingerprint recognition systems is the fingerprint matching algorithm.

A fingerprint matching algorithm compares fingerprint features and returns a matching score. The higher returned value, the greater is the fingerprints similarity. According to the problem domain, fingerprint matching algorithms are classified in two categories: fingerprint verification algorithms and fingerprint identification algorithms. The aim of fingerprint verification algorithms is to 
determine whether two fingerprints come from the same finger or not. On the other hand, fingerprint identification algorithms search a query fingerprint in a database looking for the fingerprints coming from the same finger.

Among the most popular fingerprint verification approaches we have the algorithms based on minutiae descriptors [6]. Minutiae are the points where the ridge continuity breaks, while minutiae descriptors are structures formed by one minutia and additional information. This information usually makes the descriptors invariant to rotation and translation. Minutiae descriptors are also known as local minutiae structures [10].

Minutiae descriptors can be mainly classified into ridge-based descriptors, texture-based descriptors, and neighboring minutiae-based descriptors. Ridgebased descriptors 417/39/7] associate each minutiae with the information related to the ridge where it resides. Texture-based descriptors [12 1514] enrich each minutia with the ridge orientation, the frequency information or both, at selected sampling points around it. Neighboring minutiae-based descriptors [16 11 18 5 1 13] combine each minutia with information of certain neighboring minutiae.

There is a huge diversity on both minutiae descriptors and fingerprint verification algorithms based on minutiae descriptors [10]. An important component of the algorithms based on minutiae descriptors is the alignment strategy. There are two main alignment strategies:

1. Single alignment strategy 14812 , which compares all the minutiae descriptors in both fingerprints and selects the pair that maximizes the similarity value to perform the minutiae alignment. This strategy has a low time complexity $\left(O\left(n^{2}\right)\right)$ but the accuracy is affected because a better alignment can be frequently found using a matching pair that is not the one that maximizes the similarity value.

2. Multiple alignments strategy [161118], which makes an alignment for each pair of matching minutiae descriptors. Even when many not matching pairs are used for alignment, these cases are mitigated by other correct pairs in the fingerprints. This strategy alleviates the main drawback of single alignment while increasing the time complexity to $O\left(n^{4}\right)$.

In this paper, we propose an improvement of the multiple alignments strategy. It reduces the time complexity to $O\left(n^{2}\right)$, achieving similar or better accuracy than the original strategy in most of the performance indicators, according to the evaluation protocols of FVC2004 [2].

\section{Previous Work}

In this section we describe two very different alignment strategies: the single alignment strategy followed by Tico and Kuosmanen [14] (TK), Jiang and Yau [8] (JY), and Qi et al. [12] (QYW); and the multiple alignments strategy followed by Udupa et al. [16], Parziale and Niel [11], and Zheng et al. 18]. We are interested in the first one because it is the simplest alignment strategy proposed in literature 


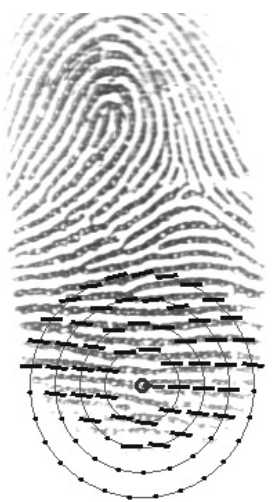

(a)

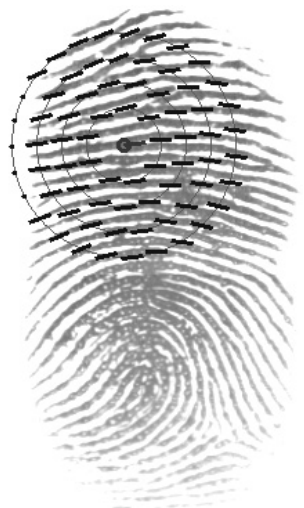

(b)

Fig. 1. Minutia pair found by the algorithm TK in fingerprints (a) 11_1 and (b) 11_3 from DB1_A in FVC2004. This is an example of a false matching minutiae pair that maximizes the similarity value in two fingerprints from the same finger.

$\left(O\left(n^{2}\right)\right.$ time complexity) and in the second one, because it overcomes some limitations of the former but increases the time complexity to $O\left(n^{4}\right)$. So, our intention is to simplify the latter to have $O\left(n^{2}\right)$ time complexity while still having high verification accuracy.

\subsection{Single Alignment Strategy}

The algorithms that follow this strategy compare the minutiae descriptors in both fingerprints, selecting the pair that maximizes the similarity value to perform the minutiae alignment. They contains three major steps:

1. Local Matching. This step matches all the minutiae descriptors in the query fingerprint with the descriptors in the template to determine local matching minutiae pairs, using a particular similarity measure. TK defines a descriptor using the estimations of the orientation values on sampling points arranged in concentric circles around the minutia. QYW uses a similar descriptor but the sampling points are organized in three lines around the minutia. The descriptor defined by JY computes several parameters from the corresponding minutia and its two nearest neighbors.

2. Global Matching. This step performs alignment according to the minutiae pair that maximizes the descriptors similarity value. Then, it uses a greedy algorithm to find the minutiae pairs that maximize the descriptors similarity while satisfying global matching constraints. QYW and JY use a 3-D bounding box to determine which local matching minutiae are consistent at the global level. TK determines the global matching minutiae from those local matching minutiae that satisfy the following constraints after alignment: the Euclidean distance between minutiae does not exceed a certain threshold, and the difference between minutiae directions does not exceed the specified threshold. 
3. Similarity Score Computation. This step computes the similarity score of the compared fingerprints. QYW and TK use the global matching minutiae together with those minutiae that fall inside the region of interest common to both fingerprints. JY uses the global matching minutiae together with all the minutiae that are present in both fingerprints.

The main drawback of this strategy is that it aligns fingerprints using a single minutiae pair (the one that maximizes the similarity value). Aligning by using the most similar minutiae pair does not guarantee to find a true matching minutiae pair (see Fig. 1). Frequently, even if the selected minutiae pair is a true matching pair, it is not necessarily the best pair to carry out fingerprint alignment.

\subsection{Multiple Alignments Strategy}

Udupa et al. [16] introduce this strategy to overcome the limitations of the single alignment strategy. This strategy performs an alignment for each pair of minutiae descriptors that match locally; so, having $n$ descriptors it must carry out $n^{2}$ alignments in the worst case scenario. For each alignment, every pair of minutiae descriptors that match locally ( $n^{2}$ in the worst case) must be examined looking for those which satisfy certain global matching constraints. This way, this strategy overcomes the problems of the single alignment strategy but with higher cost $\left(O\left(n^{4}\right)\right.$ time complexity).

In the next section, we introduce an improvement of the multiple alignments strategy which reduces the time complexity from $O\left(n^{4}\right)$ to $O\left(n^{2}\right)$ while still achieving high accuracy.

\section{Improving the Multiple Alignments Strategy}

The improved strategy is based on the assumption that most minutiae descriptors from one fingerprint correspond to their most similar descriptors from the other fingerprint. This assumption results in a lineal amount of minutia correspondences, opposite to the traditional multiple alignments strategy, which obtains a quadratic amount of minutia correspondences. Then, these correspondences are the only candidates for both local and global matching, keeping the complexity in $O\left(n^{2}\right)$. A formal description of the new strategy is the following:

1. Local minutiae matching:

(a) Let $Q$ and $P$ be the query and template fingerprint minutiae set respectively. Let $L \leftarrow\{\}$ be the set that will contain local matching minutiae pairs.

(b) For each minutiae $\mathbf{q}_{\mathbf{i}} \in Q$ select the minutia $\mathbf{p} \in P$ with higher similarity value and add the pair $\left(\mathbf{q}_{\mathbf{i}}, \mathbf{p}\right)$ to $L$.

(c) For each minutiae $\mathbf{p}_{\mathbf{j}} \in P$ select the minutia $\mathbf{q} \in Q$ with higher similarity value and add the pair $\left(\mathbf{q}, \mathbf{p}_{\mathbf{j}}\right)$ to $L$. 
2. Global minutiae matching:

(a) Sort in descendent order all matching pairs in $L$ according to the similarity value.

(b) For each $(\mathbf{q}, \mathbf{p}) \in L$, align the minutiae pairs in $L$ based on $(\mathbf{q}, \mathbf{p})$ and determine the pairs that match globally avoiding minutiae duplicity. The higher the amount of globally matching minutiae, the better the alignment.

3. Similarity score computation

The proposed strategy can be used either for improving existing algorithms or for creating new algorithms, defining the following parameters:

- Minutiae descriptor

- Similarity function to compare the minutiae descriptors

- Method to align minutiae given a reference minutiae pair

- Constraints used to consider a minutiae pair as a global matching pair

- Method to compute the similarity score.

\section{Experimental Results}

In order to evaluate our new strategy, we perfomed an experimental comparison using the databases and evaluation protocol of the FVC2004 2]. The algorithm quality is evaluated using the performance indicators EER, FMR100, FMR1000, and ZeroFMR. We also evaluate the average matching time, in milliseconds.

To compare among the strategies, we use the following algorithms:

- Algorithms TK, JY, and QYW, which use the single alignment strategy

- Algorithms UTK, UJY, and UQY, which are versions of TK, JY, and QYW adapted to the multiple alignments strategy proposed by Udupa [16]

- Algorithms MTK, MJY, and MQY, which are versions of TK, JY, and QYW adapted to our improved strategy

We can not apply our new strategy to the algorithms [1611 because they use a boolean function to compare minutiae descriptors.

We set the algorithms parameters based on the information provided in the original papers. We carry out all the experiments on a PC with an Intel Core 2 Duo processor $(2.53 \mathrm{GHz})$ and $4 \mathrm{~GB}$ of RAM.

Figure 2 shows the accuracy results of the algorithms. Table 1 shows the time results, where best results appear bolded. An analysis of the experimental results reveals the following regularities:

\section{Algorithms Based on TK}

- UTK outperforms TK according to EER and FMR100; but TK is better according to FMR1000 and ZeroFMR. Besides, TK is at least 19 times faster than UTK.

- MTK outperforms TK according to almost all the accuracy indicators. The matching time of both algorithms is similar. 


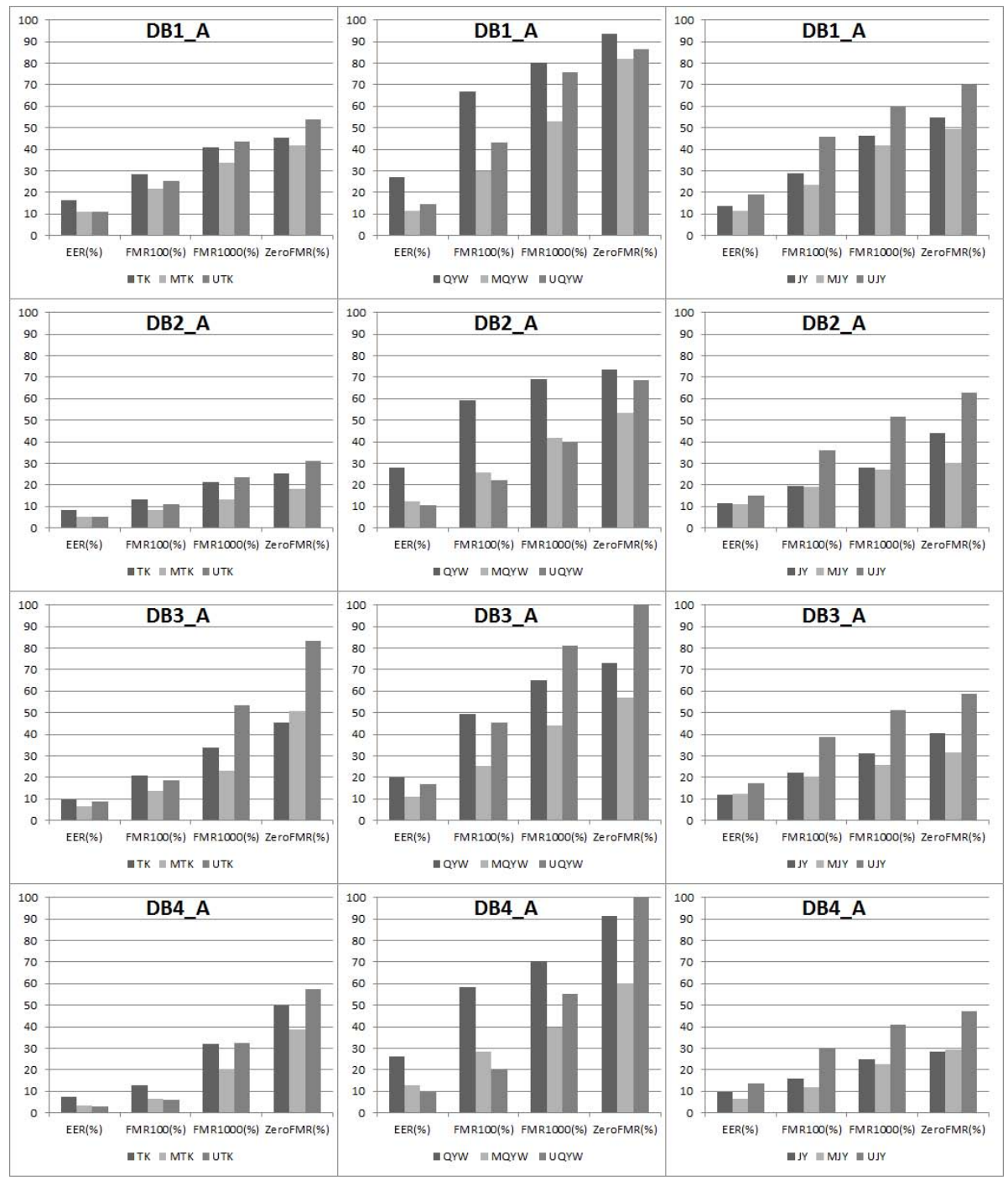

Fig. 2. Accuracy results on FVC2004 databases

Table 1. Time results on FVC2004 databases

\begin{tabular}{c|lll|lll|lll}
\hline DB / Time(ms) & TK & UTK & MTK & QYW UQYW & UQYW & JY & UJY & MJY \\
\hline DB1_A & 50.02 & 1251.17 & $\mathbf{4 9 . 8 3}$ & $\mathbf{4 6 . 3 6}$ & 1237.58 & 56.86 & $\mathbf{1 0 . 7 1}$ & 1342.89 & 28.68 \\
\hline DB2_A & $\mathbf{3 9 . 7 8}$ & 816.35 & 40.45 & $\mathbf{3 6 . 6 0}$ & 859.84 & 44.47 & $\mathbf{8 . 4 2}$ & 911.88 & 23.02 \\
\hline DB3_A & $\mathbf{7 5 . 8 4}$ & 3045.05 & 76.79 & $\mathbf{7 1 . 2 9}$ & 3133.80 & 87.44 & $\mathbf{1 5 . 6 1}$ & 3209.38 & 44.00 \\
\hline DB4_A & 41.21 & 822.65 & $\mathbf{4 1 . 0 5}$ & $\mathbf{3 7 . 0 5}$ & 932.63 & 46.38 & $\mathbf{8 . 9 8}$ & 912.15 & 23.48 \\
\hline
\end{tabular}


- MTK outperforms or is similar to UTK for all accuracy indicators; MTK is at least 19 times faster than UTK.

\section{Algorithms Based on QYW}

- UQYW outperforms QYW for most accuracy indicators; but QYW is at least 23 times faster.

- MQYW is better than QYW for all the performance indicators and it is only 1.3 times slower.

- MQYW outperforms UQYW for most accuracy indicators and it is at least 19 times faster.

\section{Algorithms Based on JY}

- UJY is worse than JY for all the performance indicators. Besides, JY is at least 100 times faster than UJY.

- MJY is better than JY for almost all accuracy indicators. JY is about 3 times faster than MJY.

- MJY outperforms UJY according to all the accuracy indicators and it is at least 39 times faster.

Experimental results reveal that the new strategy increases the accuracy of base algorithms and the multiple alignments modifications. The new strategy is at least 19 times faster than the multiple alignments modifications, while being only at most 3 times slower than base algorithms.

\section{Conclusions}

Aligning fingerprints is a crucial step in fingerprint verification. The multiple alignments strategy is an accurate solution proposed in literature, but it has a high computational cost. In this paper, we improve the multiple alignments strategy for fingerprint verification. Our strategy is based on the hypothesis that every minutiae descriptor from a fingerprint should only match the most similar in the other fingerprint. Our modifications perform multiple alignments faster than the traditional multiple alignments strategy while attaining higher accuracy.

Acknowledgment. The authors would like to thank MSc. Dania Yudith Suárez Abreu for her valuable contribution improving the grammar and style of this paper.

\section{References}

1. Cappelli, R., Ferrara, M., Maltoni, D.: Minutia cylinder-code: a new representation and matching technique for fingerprint recognition. IEEE Transactions on Pattern Analysis and Machine Intelligence 32(12), 2128-2141 (2010) 
2. Cappelli, R., Maio, D., Maltoni, D., Wayman, J.L., Jain, A.K.: Performance evaluation of fingerprint verification systems. IEEE Transactions on Pattern Analysis and Machine Intelligence 28(1), 3-18 (2006)

3. Feng, J., Ouyang, Z., Cai, A.: Fingerprint matching using ridges. Pattern Recognition 39, 2131-2140 (2006)

4. Hu, C., Yin, J., Zhu, E., Chen, H., Li, Y.: Fingerprint alignment using special ridges. In: 19th International Conference on Pattern Recognition, Tampa, Florida, USA, pp. 1-4 (2008)

5. Jain, A.K., Feng, J.: Latent fingerprint matching. IEEE Transactions on Pattern Analysis and Machine Intelligence 33(1), 88-100 (2011)

6. Jain, A.K., Feng, J., Nandakumar, K.: Fingerprint matching. Computer 43(2), 36-44 (2010)

7. Jain, A.K., Lin, H., Bolle, R.: On-line fingerprint verification. IEEE Transactions on Pattern Analysis and Machine Intelligence 19(4), 302-314 (1997)

8. Jiang, X., Yau, W.Y.: Fingerprint minutiae matching based on the local and global structures. In: 15th International Conference on Pattern Recognition, Barcelona, Spain, vol. 2, pp. 1038-1041 (2000)

9. Luo, X., Tian, J., Wu, Y.: A minutiae matching algorithm in fingerprint verification. In: 15th International Conference on Pattern Recognition, Barcelona, Spain, vol. 4, pp. 833-836 (2000)

10. Maltoni, D., Maio, D., Jain, A.K., Prabhakar, S.: Handbook of fingerprint recognition, 2nd edn. Springer, London (2009)

11. Parziale, G., Niel, A.: A Fingerprint Matching Using Minutiae Triangulation. In: Zhang, D., Jain, A.K. (eds.) ICBA 2004. LNCS, vol. 3072, pp. 241-248. Springer, Heidelberg (2004)

12. Qi, J., Yang, S., Wang, Y.: Fingerprint matching combining the global orientation field with minutia. Pattern Recognition Letters 26(15), 2424-2430 (2005)

13. Tan, X., Bhanu, B.: Fingerprint matching by genetic algorithms. Pattern Recognition 39(3), 465-477 (2006)

14. Tico, M., Kuosmanen, P.: Fingerprint matching using an orientation-based minutia descriptor. IEEE Transactions on Pattern Analysis and Machine Intelligence 25(8), 1009-1014 (2003)

15. Tong, X., Huang, J., Tang, X., Shi, D.: Fingerprint minutiae matching using the adjacent feature vector. Pattern Recognition Letters 26, 1337-1345 (2005)

16. Udupa, U.R., Garg, G., Sharma, P.: Fast and Accurate Fingerprint Verification. In: Bigun, J., Smeraldi, F. (eds.) AVBPA 2001. LNCS, vol. 2091, pp. 192-197. Springer, Heidelberg (2001)

17. Wang, X., Li, J., Niu, Y.: Fingerprint matching using orientationcodes and polylines. Pattern Recognition 40(11), 3164-3177 (2007)

18. Zheng, J.D., Gao, Y., Zhang, M.Z.: Fingerprint matching algorithm based on similar vector triangle. In: 2nd International Congress on Image and Signal Processing, CISP 2009, pp. 1-6 (2009) 